\title{
Criminologie
}

\section{La criminalité environnementale transnationale : aux grands maux, les grands remèdes?}

\section{Amissi M. Manirabona}

Volume 47, numéro 2, automne 2014

Criminalité et police transnationales : une perspective critique

URI : https://id.erudit.org/iderudit/1026732ar

DOI : https://doi.org/10.7202/1026732ar

Aller au sommaire du numéro

\section{Éditeur(s)}

Les Presses de l’Université de Montréal

ISSN

0316-0041 (imprimé)

1492-1367 (numérique)

Découvrir la revue

Citer cet article

Manirabona, A. M. (2014). La criminalité environnementale transnationale : aux grands maux, les grands remèdes ? Criminologie, 47(2), 153-178.

https://doi.org/10.7202/1026732ar
Résumé de l'article

Cette étude propose la façon dont la communauté internationale devrait traiter la criminalité environnementale transnationale. Pour y arriver, l'auteur définit d'abord ce phénomène et discute de son ampleur. La démarche consiste à faire comprendre cette forme de criminalité afin d'amener les parties intéressées à envisager les moyens appropriés pour la combattre. Ensuite, l'article rappelle l'inexistence des moyens de lutte à la hauteur de la gravité de ce fléau et soutient par la suite que l'ampleur et les caractéristiques de celui-ci méritent que la communauté internationale se mobilise dans son ensemble. Plus précisément, l'auteur soutient qu'en dehors du renforcement des mécanismes de lutte sur le plan national, de l'amélioration de la coopération judiciaire et de la reconnaissance mutuelle des décisions de justice, la création d'un tribunal international pénal pour l'environnement serait une option idéale. 


\title{
La criminalité environnementale transnationale: aux grands maux, les grands remèdes?
}

\author{
Amissi M. Manirabona ${ }^{1}$ \\ Professeur adjoint \\ Faculté de droit, Université de Montréal \\ melchiade.manirabona@umontreal.ca
}

\begin{abstract}
RÉSUmÉ - Cette étude propose la façon dont la communauté internationale devrait traiter la criminalité environnementale transnationale. Pour y arriver, l'auteur définit d'abord ce phénomène et discute de son ampleur. La démarche consiste à faire comprendre cette forme de criminalité afin d'amener les parties intéressées à envisager les moyens appropriés pour la combattre. Ensuite, l'article rappelle l'inexistence des moyens de lutte à la hauteur de la gravité de ce fléau et soutient par la suite que l'ampleur et les caractéristiques de celui-ci méritent que la communauté internationale se mobilise dans son ensemble. Plus précisément, l'auteur soutient qu'en dehors du renforcement des mécanismes de lutte sur le plan national, de l'amélioration de la coopération judiciaire et de la reconnaissance mutuelle des décisions de justice, la création d'un tribunal international pénal pour l'environnement serait une option idéale.
\end{abstract}

MOTS CLÉS • Environnement, crimes, transnational, dommage, gravité.

\section{Introduction}

Même s'il est souvent affirmé que c'est depuis des siècles que les activités humaines ont changé la nature de notre environnement et sa biodiversité (White, 2010), la criminalité environnementale n'est devenue un réel problème que depuis le début du développement industriel. Alors que l'action humaine n'avait guère posé de sérieuses difficultés à l'environnement, la montée en puissance de l'industrialisation a abouti

1. Université de Montréal, Faculté de droit, Pavillon Maximilien-Caron, C. P. 6128, succursale Centre-ville, Montréal, (Québec), Canada, H3C 3J7. 
à la production des déchets toxiques qui ont progressivement détruit l'environnement et menacé la vie et la santé humaines. En outre, la mondialisation ayant suivi l'ouverture des frontières nationales aux capitaux étrangers ainsi que la rapidité et la facilité du transport international ont entraîné une progression rapide des activités économiques transnationales légales et illégales, y compris la criminalité environnementale. La mondialisation a favorisé l'ouverture de nouvelles opportunités criminelles destinées à satisfaire l'augmentation exponentielle de nouvelles demandes en biens de consommation situés loin des frontières nationales (Elliot, 2012). À cela s'ajoutent les facilités de communication et de voyage à travers les États ainsi que la perméabilité des frontières, qui n'ont fait qu'accentuer le marché global des produits licites et illicites. Les systèmes d'intégration régionale visant à lever au maximum les formalités de voyages ont rendu faciles les déplacements des individus et des biens de services et de consommation (Elliot, 2012). L'accroissement du volume des importations et des exportations a rendu ardu le contrôle des cargaisons et plus facile la dissimulation des produits frauduleux. Pour sa part, le développement des nouvelles technologies d'information et de communication a permis aux trafiquants de dissimuler ou de brouiller les traces de leurs transactions alors que le transport dans des conteneurs permet de cacher les produits illégaux à l'intérieur de ceux légaux (United Nations Environment Programme [UNEP], 2013).

La criminalité environnementale transnationale (CET) est en croissance exponentielle en termes de variété, de volume et de valeur financière. Son impact va plus loin que la simple destruction de ressources naturelles, de la biodiversité et de l'habitat. Il affecte la sécurité humaine en alimentant et en entretenant les conflits, en compromettant la primauté du droit, la bonne gouvernance et la jouissance des droits fondamentaux comme le droit à la vie et à la sécurité, à l'alimentation, à l'eau potable, à la protection contre les intempéries, etc. (Agnew, 2012). Certains groupes de criminels environnementaux ont recours aux mêmes méthodes sophistiquées ainsi qu'aux mêmes réseaux que les trafiquants d'êtres humains, de stupéfiants, d'armes ou autres produits de contrebande (Scanlon, 2012).

Or, il demeure difficile pour le public d'appréhender la véritable nature de la CET ainsi que sa dangerosité. Les impacts réels de la CET sont également inconnus ou inconcevables en raison du manque de capacité et de volonté politiques pour la traquer et la combattre. De 
l'autre, il n'existe pas d'instrument international réellement destiné à la lutte contre la CET dans son ampleur. Une grande partie de la CET n'est régie par aucun traité. Par ailleurs, les traités qui ont été adoptés pour régir certains aspects de la CET ne comportent pas de mesures efficaces de mise en œuvre afin de dissuader les criminels environnementaux. Les quelques instruments comportant des dispositions criminelles se contentent de renvoyer aux États parties pour l'adoption des mesures concrètes visant à punir les contrevenants. Or, la préoccupation de plusieurs États touchés est loin d'être la CET, ce qui fait que plusieurs États ne criminalisent pas cette forme de conduite ou n'ont pas de moyens matériels et humains nécessaires pour réprimer les auteurs des violations.

Tel que le résume Interpol, la lutte efficace contre la CET fait face à de nombreux défis, à savoir l'incohérence des stratégies, le manque de coopération, la faiblesse de mise en œuvre des lois, le manque d'engagement politique, la corruption endémique, le manque d'avancée et de délais ainsi que le manque d'analyse et de compréhension (Interpol, 2013). Pour sa part, l'Office des Nations Unies contre la drogue et le crime est convaincu que les obstacles à la lutte, par les États, contre le phénomène de la CET sont dus à l'absence d'intérêt pour les crimes survenus à l'étranger, au manque de compréhension des phénomènes criminels locaux, à l'insuffisance de la sensibilisation, au non-respect des instruments juridiques internationaux ainsi quaux perceptions contradictoires sur le plan mondial et régional. L'Office soutient ainsi que le développement et le renforcement des mécanismes de lutte sur le plan supranational aideraient à surmonter ces obstacles (Office des Nations Unies contre la drogue et le crime [ONUDC], 2012a).

Comme la situation sur le terrain le prouve, toute initiative de lutte efficace contre cette forme de criminalité peut d'abord se heurter à la complexité du phénomène. Alors que certains crimes environnementaux sont de nature opportuniste et occasionnelle, un grand nombre d'entre eux s'opèrent en réseaux criminels organisés. Il s'agit en réalité d'une gamme complexe d'opérateurs à l'intérieur et à l'extérieur des pays impliquant plusieurs catégories d'objets, de participants et de criminels ayant recours aux méthodes bien rodées leur permettant d'échapper à l'application de la loi par l'effacement des traces et la falsification d'informations sur leur véritable nature ou origine (Elliot, 2012). Certains de ces réseaux criminels sont plus puissants que plusieurs États et leur capacité organisationnelle et financière est comparable à celle 
des industries puissantes. À titre d'exemple, il est estimé que le commerce illégal du bois rapporte annuellement près de 100 milliards de dollars aux trafiquants et que les régions les plus touchées par le phénomène sont l'Amazonie, l'Afrique centrale et l'Asie du Sud-Est dont 50 à $90 \%$ du commerce du bois est illégal (UNEP \& Interpol, 2012).

Par ailleurs, il n'est pas étonnant que la CET trouve un ancrage important dans des pays en développement où les mécanismes de mise en œuvre des lois sont faibles, que ce soit par manque de ressources matérielles, financières et humaines adéquates ou par corruption administrative ou pénurie d'informations. En effet, comme le souligne Williams, la CET est justement caractérisée par le fait que les criminels visent des régions ou des pays faiblement surveillés ou non réglementés afin que des crimes se commettent à moindre coût tout en procurant un maximum possible d'avantages (Williams, 1996).

Le but de cet article est de souligner l'ampleur de la CET d'un côté et, de l'autre, l'inexistence des moyens de lutte à la hauteur de la gravité de ce fléau. Il est impératif que la CET ne continue pas à s'intensifier au détriment de la vie, de la santé et du bien-être socioéconomique de l'humanité. Après avoir passé en revue les différentes conséquences engendrées par la CET, l'article relève que l'ampleur et les caractéristiques de celles-ci méritent que la communauté internationale se mobilise dans son ensemble. À ce sujet, la première section de cet article explore les aspects conceptuels de la CET alors que la deuxième section est réservée à l'ampleur des dommages causés par le phénomène. La démarche consiste à faire comprendre cette forme de criminalité afin d'amener les parties intéressées à envisager les moyens appropriés pour la combattre. La dernière section aborde la nécessité d'internationaliser la lutte contre le phénomène de la CET en mettant au jour la faiblesse des moyens existants et en soulignant l'importance de les renforcer, par un régime autonome de répression internationale.

\section{Aspects conceptuels de la criminalité environnementale transnationale}

En 1994, la résolution 1994/15 du Conseil économique et social des Nations Unies sur le rôle du droit pénal dans la protection de l'environnement faisait déjà état du caractère transnational de certaines atteintes environnementales (Organisation des Nations Unies [ONU], 1994). En 1995, lorsque les Nations Unies ont élaboré la liste de 
18 crimes transnationaux, la CET y était incluse (UNODC, 1995). En s'appuyant sur la description des atteintes environnementales faite par Interpol (2012), on peut soutenir que la CET consiste en actes ou omissions aux dimensions transfrontalières ou globales qui violent la loi et qui peuvent entraîner des poursuites et des sanctions criminelles. La lecture attentive de la Convention des Nations Unies contre la criminalité transnationale organisée permet d'y apporter une précision importante: il s'agit des infractions commises dans plus d'un État ou dans un seul État mais avec une partie substantielle de leur préparation, de leur planification, de leur conduite ou de leur contrôle qui a lieu dans un autre État. Elles peuvent aussi être commises dans un État mais impliquer un groupe criminel organisé qui se livre à des activités criminelles dans plus d'un État, ou commises dans un État avec des effets substantiels dans un autre État (art. 3).

Les crimes environnementaux transnationaux sont par exemple la pollution de l'air, de l'eau et des terres ainsi que le trafic illégal des animaux sauvages, des plantes ou de matières appauvrissant la couche d'ozone, le commerce et le rejet de déchets ou de matières toxiques dans l'environnement, l'abattage illégal d'arbres, le commerce de l'ivoire d'éléphants et la pêcherie illégale, commis au mépris des législations nationales et du droit international. Dans son rapport Global Response to Transnational Organized Environmental Crime, Gosling du Global Initiative against Transnational Organized Crime fait mention d'un certain nombre de crimes environnementaux qui recoupe plusieurs de ceux mentionnés par Interpol, y compris l'extraction illégale et à grande échelle des ressources naturelles (Gosling, 2013). À cela s'ajoutent les mouvements transnationaux des industries polluantes des pays développés vers les pays pauvres, ainsi que la surpêche illégale dans des océans (White, 2011).

Interpol rapporte aussi de nouvelles formes de crimes environnementaux comme le commerce illégal du carbone et la gestion illégale de l'eau (Interpol, 2012). La criminalité environnementale impliquant le trafic des émissions de carbone est un exemple d'une CET d'un type récent mais qui est déjà très attrayante pour les organisations criminelles transnationales. En effet, le Guide Interpol sur cette forme de criminalité révèle que le commerce des émissions de carbone est le marché de matières premières dont la croissance est la plus rapide et que sa valeur tourne autour de 176 milliards de dollars. À la différence des marchés traditionnels, aucune marchandise physique n'y est échangée mais 
uniquement des «crédits carbone» correspondant à des émissions de dioxyde de carbone (Interpol, 2013). C'est précisément la difficulté d'estimer la valeur de ce marché, les sommes considérables impliquées et l'absence de tout contrôle, qui l'exposent à l'assaut des groupes criminels.

Comme on peut le constater, la définition institutionnelle de la CET concerne principalement la violation des conventions internationales et/ou des droits nationaux. Or, les criminologues verts préfèrent une définition plus ou moins large qui met l'accent sur le préjudice subi en considérant la CET comme un acte ou une omission qui nuit à l'être humain, à l'environnement et aux animaux, indépendamment de leur légalité ou illégalité (White, 2011). En effet, d'un côté, certaines atteintes environnementales ne sont régies par aucun instrument international et, de l'autre, les dispositions pénales nationales visant la protection de l'environnement sont plutôt rares dans plusieurs pays (Skinnider, 2011). Par ailleurs, on sait bien que certaines actions non criminalisées causent plus de dommages aux victimes humaines, animales ou végétales que les actions criminalisées (Lynch et al., 2010). D'où l'importante de définir la CET en considérant le préjudice subi par les victimes plutôt qu'en se contentant des catégories préétablies par la loi (Hall, 2013; White, 2011). Dès lors, on peut définir la CET comme une conduite impliquant des acteurs situés dans au moins deux pays et qui crée un préjudice environnemental dans au moins un pays, qu'elle soit en violation ou non d'une loi existante. Cette conduite vise à procurer des avantages financiers ou à maintenir ou étendre la puissance des délinquants qui sont généralement des acteurs non étatiques agissant comme producteurs ou récepteurs (Mason, 2008). Elle est, enfin, menée avec préméditation, intention, connaissance ou aveuglement volontaire (Gilbert \& Russel, 2002).

Il sied par ailleurs de mentionner que si la CET est souvent le fruit d'une entreprise entièrement criminelle, elle peut aussi découler ou se greffer aux activités licites. En outre, des activités tout à fait légales peuvent s'avérer par la suite nocives à l'environnement et à la santé des individus exposés comme le fait pour une entreprise de polluer l'environnement par une exploitation anarchique des mines dans un État étranger (Mason, 2008). 


\section{Aperçu de l'ampleur des dommages causés par la criminalité environnementale transnationale}

Le coût de la CET se résume en atteintes graves à la santé, à la sécurité et à la vie des individus et des espèces sauvages, ainsi qu'à la pollution sévère à l'origine de la disparition de l'habitat, de la biodiversité et du changement climatique. Plusieurs cas de criminalité environnementale sont graves et comparables à ceux des crimes traditionnels contre la vie et la sécurité des individus, y compris les crimes contre l'humanité (Manirabona, 2011a). La CET est au centre d'un très grand nombre de dommages, y compris les menaces d'extinction des espèces sauvages, la dégradation de l'environnement local ou global, la détérioration de la santé des générations présentes et à venir, etc. (UNEP, 2013). Il importe de parler de l'ampleur de la CET selon qu'elle s'exprime dans le cadre de la criminalité organisée ou selon qu'elle est occasionnée par des activités économiques légales.

\section{Ampleur de la CET survenue dans le cadre de la criminalité organisée}

En premier lieu, mentionnons l'abattage massif et illégal d'espèces d'arbres naturels à l'origine d'importantes déforestations et de la destruction de l'habitat et des espèces protégés. Il a été documenté que cette forme de CET qui cause la diminution des espèces sauvages rompt les équilibres biologiques et est à l'origine de la dégradation sévère de l'environnement (Elliot, 2012). D'après Interpol, l'extinction des espèces par le braconnage ou l'abattage illégal des arbres peut conduire à la dégradation de la qualité de l'écosystème dont dépend notre existence ainsi que l'accélération du réchauffement de la planète et du changement climatique. Par ailleurs, il a été rapporté que la dégradation des forêts prive les populations autochtones de leur mode de vie, cause des problèmes écologiques comme la montée des eaux et les changements climatiques, considérant que $20 \%$ des gaz à effet de serre proviennent de la déforestation (O’Hear, 2004).

Le commerce illégal des substances détruisant la couche d'ozone est quant à lui à l'origine de plusieurs maux, y compris les cancers de la peau, la cataracte, l'affaiblissement du système immunitaire chez les humains et les animaux ainsi que la réduction de la productivité des plantes. À un moment donné, la vie sur terre pourrait être pratiquement 
impossible dans le cas où la couche d'ozone qui nous protège contre les rayons solaires serait sérieusement détruite.

Le déversement des déchets dangereux dans l'environnement contribue à la contamination des terres, de l'eau, de la santé tout en augmentant les impacts négatifs sur la chaîne alimentaire (Elliot, 2012). Comme le précise Interpol, en plus de représenter un danger pour la santé humaine, les rejets illicites de déchets dans l'eau, dans l'air et dans le sol peuvent avoir de graves conséquences sur les moyens de subsistance de toute une population, détruire des emplois et faire perdre aux biens immobiliers une partie de leur valeur, menaçant ainsi la viabilité économique à long terme de même que la stabilité nationale (Interpol, 2014).

Par ailleurs, le déversement de 581 tonnes de déchets toxiques opéré en 2006 en Côte d'Ivoire par une entreprise multinationale de droit anglais, Trafugira, a entraîné, entre autres, des dizaines de décès ainsi qu'une centaine de milliers de victimes blessées ou physiquement touchées d'une manière ou d'une autre. Les victimes ont notamment souffert de problèmes d'irritation des narines, de douleurs thoraciques, de difficultés respiratoires, d'essoufflement, de toux, de brûlures, de douleurs et autres infections des yeux, de maux de gorge, de douleurs abdominales, de nausées et de vomissements, d'irritations et sensations de brûlure et des démangeaisons graves de la peau, etc. (Manirabona, 2011b). En outre, on a relevé des atteintes graves à l'environnement, notamment les sites hautement pollués qui mettront plusieurs années avant d'être totalement décontaminés.

En ce qui concerne les conséquences de la CET sur les espèces sauvages et la biodiversité, la situation est également préoccupante. Par exemple, on estime qu'au cours de l'année 2013, plus de 1000 rhinocéros ont été tués en Afrique du Sud (Agence France-Presse [AFP], 2014). Une tendance semblable est observable, quoiqu'à un moindre degré, dans certains pays de l'Afrique australe comme le Zimbabwe, le Malawi, l'Angola, le Botswana, le Mozambique et la Namibie.

Pour ce qui est des éléphants, il a été rapporté que, pour la seule année 2012, près de 30000 d'entre eux ont été tués en Afrique. En moyenne, entre 22 et 25000 éléphants sont tués chaque année dans cette région, soit plus de 60 par jour de sorte qu'il ne resterait qu'un demi-million de bêtes contre 1,2 million il y a trente ans (TRAFFIC, 2013). Au Kenya seulement, le braconnage serait à l'origine de la réduction du nombre d'éléphants de 167000 à 35000 en une ving- 
taine d'années. Toutefois, c'est en Afrique centrale que la situation semble se détériorer le plus avec un taux d'abattage d'éléphants trois fois plus élevé qu'ailleurs sur le continent (TRAFFIC, 2013). Selon les Nations Unies, au Gabon, 11000 éléphants ont été illégalement abattus sur une période de neuf ans entre 2004 et 2013, dans le seul Minkebe Park au nord-est du pays. Au Cameroun, 300 éléphants furent tués au courant des mois de novembre et décembre 2012, tandis qu'au Tchad, 86 éléphants (parmi lesquels il y avait 33 femelles gravides) furent décimés en une semaine seulement au cours du mois de mars 2013 (ONU, 2013). La situation des éléphants en Afrique est tellement préoccupante que dans quelques décennies, on craint une extinction totale des plus grands mammifères terrestres de la planète (Union internationale pour la conservation de la nature [UICN], 2013).

\section{Ampleur de la CET survenue dans le cadre des activités économiques licites}

Certaines activités économiques légales peuvent déboucher sur la CET lorsqu'elles sont menées sans aucun égard à l'environnement. À ce sujet, on n'a qu'à penser aux cinq mille morts et le demi-million de blessés ou empoisonnés à la suite de la catastrophe de Bhopal survenue en Inde en 1986 à cause du manque d'entretien adéquat de l'usine. À ce jour, les bébés de cette ville continuent de naître avec des malformations congénitales près de trente ans après le désastre (Amnesty International, 2004). Un autre exemple patent est le récent déversement du pétrole dans le golfe du Mexique par la plateforme pétrolière Deepwater Horizon de l'entreprise BP qui, en plus de tuer sur-le-champ 11 personnes et d'en blesser 17 autres, a entraîné des problèmes de santé pour plusieurs milliers de personnes exposées (Casselman, 2011). À cela s'ajoute la contamination d'une immense étendue des côtes américaines allant de la Louisiane à la Floride en passant par le Mississippi et l'Alabama. Une estimation provisoire ayant suivi la catastrophe a fait état d'un déversement de 184 millions de barils de pétrole sur plus de 6400 kilomètres carrés (Oxford Economics, 2010). Il est également fait mention d'une perte de 1200 emplois et d'un éventuel coût de 22,7 milliards de dollars sur trois ans (Oxford Economics, 2010).

Du côté européen, il sied de signaler les cas de marées noires causées par le navire Erika en 1999 et le pétrolier Prestige en 2002 qui constituent les pires incidents environnementaux jamais connus dans la 
région. Les impacts réels de ces catastrophes demeurent par ailleurs impossibles à déterminer avec précision (Bossy, 2012).

En Afrique, les cas de la CET les plus emblématiques restent les actes de pollution causés par les entreprises pétrolières au Nigéria, le déversement des déchets toxiques en Côte d'Ivoire, l'exploitation anarchique des minerais au Mali, etc. Au sujet du Nigéria par exemple, l'ONG Amnesty International a, en 2009, rendu public un rapport sur le Delta du Niger relevant les violations massives des droits fondamentaux de la personne commises lors des activités liées à l'exploitation du pétrole par des entreprises multinationales, notamment Shell. Ces violations concernent en particulier le droit à un niveau de vie adéquat, y compris le droit à l'alimentation à la suite de la pollution des eaux servant à la pêche et des terres agricoles qui constituaient une source d'alimentation pour la majorité de la population de la région. L'organisation mentionne aussi les violations du droit de gagner sa vie par le travail, du droit à l'eau potable, du droit à la santé et du droit à la justice pour la population victime des violations (Amnesty International, 2009).

\section{Liens entre la CET, l'insécurité et la mauvaise gouvernance}

Comme nous l'avons déjà souligné, certaines formes de la CET peuvent directement causer la mort ou des blessures graves aux personnes exposées. Les activités économiques dommageables à l'environnement peuvent aussi donner lieu à la commission de meurtres et autres atteintes à la vie lorsque les milices défendant les sites où ont lieu ces activités s'en prennent à la population qui s'y oppose (Choc c. HudBay, 2013). À l'inverse, la sécurité humaine peut être directement menacée par la criminalité environnementale en raison des attaques violentes menées contre les individus travaillant à la défense de certains sites stratégiques ou des populations vivant autour de ces sites. Ainsi, il a été rapporté que des membres des communautés rurales ou des agents de protection de certains parcs sont victimes d'attaques armées organisées et menées par les criminels à la recherche d'endroits pour déverser les déchets dangereux ou à la recherche d'espèces animales sauvages à braconner et à commercialiser illégalement. En Afrique, ces attaques prennent une ampleur sans précédent à l'heure actuelle en raison de l'implication des groupes rebelles et terroristes dans le braconnage des éléphants et des rhinocéros pour financer leurs activités criminelles (ONU, 2013). Dans la mesure où l'ivoire des éléphants et les cornes des rhinocéros sont 
vendus plus cher que l'or ou la cocaïne sur le marché noir dans certaines régions de l'Asie, les groupes terroristes s'y investissent en masse avec des équipements parfois plus puissants que ceux dont disposent les forces de sécurité de plusieurs pays. C'est le cas du groupe terroriste somalien Al-Shabaab, dont près de la moitié des fonds illégalement obtenus proviennent du braconnage des animaux dans les forêts de l'Afrique de l'Est, particulièrement au Kenya (IRIN, 2013). La rébellion de l'Ouganda, la LRA, est également connue pour financer ses activités de violence par le braconnage des éléphants en République démocratique du Congo et en République centrafricaine (ONU, 2013). Il en est ainsi des groupes armés rebelles du Soudan, les Janjaweed, qui sont très actifs dans le braconnage au Tchad (Elliot, 2012).

Par ailleurs, il a été documenté que la pauvreté et la rareté des ressources de première nécessité, comme la nourriture, l'eau, l'habitation, dues à la dégradation environnementale, ont le potentiel d'augmenter les conflits sociaux non seulement sur le plan des sous-groupes de population, mais aussi sur le plan national et interétatique (Agnew, 2012). Ces conflits risquent de s'intensifier dans les pays pauvres où il est difficile ou même impossible de s'adapter aux changements environnementaux alors qu'une importante partie de la population active n'a aucun emploi. De plus, Mares (2010) décrit le lien de cause à effet entre la dégradation des terres cultivables et le déplacement forcé de certaines populations africaines qui, en plus, sont victimes du trafic d'êtres humains (Jasparro \& Taylor, 2008).

En dehors de ces conséquences essentiellement basées sur la santé et la sécurité humaines, la CET cause d'autres conséquences en termes de gouvernance et de sécurité. La CET peut par exemple engendrer diverses formes de criminalité économique comme la corruption, le blanchiment d'argent, l'évasion fiscale, le trafic d'êtres humains, le vol d'identité ou d'informations personnelles, le vandalisme, la fraude des documents administratifs, etc. À titre d'exemple, le Guide Interpol sur la criminalité liée au commerce des émissions de carbone dans ce domaine révèle que les criminels se livrent à l'escroquerie, aux délits d'initiés, aux détournements ainsi qu'au blanchiment de fonds, et aux infractions relevant de la cybercriminalité (Interpol, 2013).

En définitive, la CET affaiblit la gouvernance démocratique des États et compromet ainsi la primauté du droit en encourageant les fonctionnaires publics à violer la loi au détriment de l'intérêt général. Là où la corruption est très répandue, les États se retrouvent facilement en 
position opposée à leur rôle traditionnel de pourvoyeur et garant du bien-être social pour se transformer en complices des organisations criminelles au service des intérêts privés (Elliot, 2012). En échappant à tout contrôle et en évitant les impôts et taxes, la CET prive par ailleurs les États d'importantes ressources financières qui devraient servir à l'amélioration des conditions sociales des gouvernés (OCDE, 2012).

L'ampleur de la CET requiert que les autorités concernées adoptent des mesures de contrôle et/ou les mettent en œuvre de façon efficace afin qu'elle ne continue plus à fragiliser l'environnement et les populations locales déjà en difficulté. Mais le grand défi est de pouvoir établir correctement ces mesures et la manière dont elles doivent être exécutées. La deuxième section de cet article tentera de relever ce défi.

\section{Vers l'internationalisation de la répression de la CET}

\section{Inefficacité de l'actuel contrôle de la CET sur le plan international}

Malgré son ampleur, la CET n'est pas encore considérée comme une criminalité préoccupante pour la communauté internationale. Cela s'explique par le fait qu'aucun instrument international n'a encore été adopté pour s'occuper du phénomène dans sa globalité. Les principaux instruments des Nations Unies comme la Convention contre la criminalité transnationale organisée (CNUCTO) et la Convention contre la corruption ne se préoccupent pas davantage de la CET (Elliot, 2012). Le préambule de la CNUCTO se contente de mentionner que l'Assemblée générale des Nations Unies est fermement convaincue que cette convention «constituera un outil efficace et le cadre juridique nécessaire de la coopération internationale dans la lutte contre, notamment, des activités criminelles telles que (...) le trafic illicite des espèces de faune et de flore sauvages menacées d'extinction, les atteintes au patrimoine culturel (...)». Par conséquent, le phénomène de la CET est mal connu et mal réglementé, ce qui fausse le fonctionnement des mécanismes de coopération interétatique chargés de lutter contre le phénomène (Elliot, 2012).

Toutefois, alors que plusieurs aspects de la CET ne sont régis par aucun instrument, certains domaines sont réglementés par quelques conventions et traités internationaux. Il s'agit en particulier du domaine 
des déchets dangereux et de celui des espèces sauvages rares. À ce sujet, il y a d'abord le Protocole de Montréal relatif à des substances qui appauvrissent la couche d'ozone (1987) qui a pour objectif de protéger la couche d'ozone contre le risque d'appauvrissement en mettant en œuvre un système d'autorisation des importations et des exportations de certaines substances. Ensuite vient la Convention de Bâle adoptée en 1989 afin de faciliter le contrôle, au niveau international, des mouvements transfrontières et l'élimination des déchets dangereux pour la santé humaine et l'environnement. Les articles 4 et 6 de cette convention imposent des conditions strictes aux mouvements transfrontières des déchets dangereux en exigeant notamment une information suivie d'une acceptation expresse des autorités de destination ainsi qu'en interdisant le commerce de ces déchets avec les États sans capacité de les traiter ou ceux non parties. L'article 4(3) de la Convention prévoit que: «Les Parties considèrent que le trafic illicite de déchets dangereux ou d'autres déchets constitue une infraction pénale.» De la même façon, la Convention sur la diversité biologique (CDB) a été adoptée en 1992 à Rio afin de contribuer à la conservation de la biodiversité, et à l'utilisation et au partage durable, juste et équitable des avantages découlant de son exploitation.

Par ailleurs, la Convention de Rotterdam sur la procédure de consentement préalable en connaissance de cause réglemente les exportations / importations de 43 produits et catégories de produits chimiques dangereux, à majorité des pesticides. Cette convention adoptée en 1998 établit une procédure de consentement concernant l'importation des produits chimiques dangereux comme l'amiante.

Enfin, il y a la Convention de Stockholm adoptée en 2001 et visant à réglementer la circulation internationale de certains polluants organiques persistants (POP) comme les polychlorobiphényles (PCB). Les objectifs de cette convention visent à éliminer ou à restreindre la production et l'utilisation de tous les POP produits intentionnellement comme les matières chimiques industrielles et les pesticides, ainsi que ceux produits de façon non intentionnelle, comme les dioxines et les furanes.

Relativement au commerce des espèces sauvages protégées, deux conventions sont très importantes, à savoir la Convention sur le commerce international des espèces de faune et de flore sauvages menacées d'extinction (CITES, 1973) ainsi que la Convention sur la conservation des espèces migratrices appartenant à la faune sauvage (CMS, 1979). 
Mais ces différents instruments comportent des faiblesses énormes. Tout d'abord, il sied de souligner qu'ils ne couvrent pas une large gamme de la CET telle qu'elle est définie. En effet, d'importants domaines de la CET comme la pêche illégale, le rejet des déchets électroniques dans l'environnement, la pollution transnationale ou l'abattage illégal d'arbres, ne sont couverts par aucun traité ou accord. Ensuite, certains des instruments applicables à la CET sont très vieillissants et ne reflètent plus les réalités actuelles de la CET. À titre d'exemple, les parties au Protocole de Montréal de 1987 relatif à des substances qui appauvrissent la couche d'ozone n'avaient pas prévu que sa mise en œuvre pourrait générer un marché illégal (Elliot, 2012).

Par ailleurs, les instruments sur les matières dangereuses prévoient des listes des produits exclus de la circulation ou sujets aux autorisations administratives que la lourdeur du processus ne permet pas de mettre à jour rapidement. Ensuite, certains États peuvent respecter à la lettre les listes établies alors que d'autres peuvent décider de ne pas réglementer un type de produit pourtant mentionné sur la liste. Les conséquences sont dès lors énormes, comme l'a prouvé l'incapacité de la Convention de Bâle à empêcher que des centaines de tonnes de déchets toxiques soient exportées vers les pays pauvres ou peu réglementés.

Parmi les autres faiblesses communes de ces conventions et traités relatifs à la CET, il y a le fait qu'ils n'interdisent pas la circulation, à travers les frontières internationales, d'un grand nombre de matières dangereuses. Ces instruments ne font que soumettre cette circulation aux règles et conditions que les États déterminent, ce qui complique davantage sa mise en œuvre. Certes, certaines régions avaient tenté d'adopter des traités plus robustes comme la Convention de Bamako en Afrique, adoptée en 1991, et visant la prohibition pure et simple de l'importation de toute forme de déchets dangereux. Mais à l'instar des autres instruments portant sur la CET, cette convention souffre d'un manque de consensus parmi les États de la région ainsi que d'une absence totale de mécanismes de contrôle de sa mise en œuvre. Par conséquent, des centaines de tonnes de déchets hautement toxiques ont été exportées et déversées en Côte d'Ivoire en 2006 malgré l'existence de cette convention, (Manirabona, 2011b).

Par ailleurs, soulignons le fait que les traités relatifs à la CET ne s'appliquent qu'aux États parties. Dès lors, les dispositions de ces traités deviennent fragilisées lorsque certains États parties sont laxistes ou complices des auteurs de la CET. De même, certains de ces instruments 
imposent aux États parties l'obligation d'adopter des lois nationales pour s'attaquer à leur violation, mais aucun mécanisme de sanction n'est prévu lorsque ceux-ci ne le font pas. Et même si des lois nationales étaient adoptées, leur mise en œuvre demanderait des moyens financiers supplémentaires que ces États ne possèdent pas nécessairement ou ne sont pas prêts à débourser. Pour plusieurs États, la lutte contre la CET ne constitue pas leur priorité, surtout en période de crise économique. La plupart des États, y compris ceux développés comme le Canada, sont de plus en plus préoccupés par leur développement économique plutôt que par la CET qui est par ailleurs mal connue.

Il existe bien évidemment quelques mécanismes régionaux et sousrégionaux de mise en œuvre des normes visant à lutter contre certaines formes de la CET. C'est l'exemple de l'ASEAN Wildlife Enforcement Network, du Horn of Africa Wildlife Enforcement Network; Central African Wildlife Enforcement Network, de la Lusaka Agreement Task Force (LATF), Rhino and Elephant Security Group of Southern Africa (SADC), ou du Wildlife Enforcement Network for Southern Africa. Mais ces réseaux ne se limitent qu'à certains domaines ciblés de la CET, généralement l'abattage des éléphants et des rhinocéros. En outre, même s'ils constituent une avancée notable dans la lutte contre une partie de la CET, ces mécanismes manquent de coordination et d'appui matériel, financier et humain de la part des États impliqués, notamment en Afrique et en Asie, qui sont pourtant des régions particulièrement touchées par le phénomène. Le manque de soutien permanent des États à ces réseaux peut être expliqué par le fait que la plupart d'entre eux ont été mis en place sous l'influence des organisations internationales, généralement occidentales comme le WWF et l'UICN. Or, devant les défis de la pauvreté sans cesse grandissante, il est possible que la protection de la faune et de l'environnement en général ne soit pas perçue comme une priorité nécessitant qu'on y consacre la moindre portion du peu de ressources dont disposent plusieurs pays en développement (Wright, 2011).

\section{Caractère insatisfaisant du contrôle de la CET par les mécanismes nationaux}

De tout temps, il appartient à l'État où les crimes sont commis d'en punir les auteurs. Toutefois, la faiblesse des institutions étatiques ainsi que la pression visant à attirer les investissements font que certains 
auteurs de la CET échappent à l'application de la loi (Clapp, 2005). Par ailleurs, comme le suggère sa définition, la CET dépasse le cadre d'un seul État. Il s'agit d'une forme de criminalité qui non seulement s'étend sur plus d'un État mais aussi tend à se produire sur des territoires qui sont peu ou pas réglementés. Ainsi, les auteurs vont préférer commettre des actes apparemment légaux à l'étranger alors que ceux-ci seraient illégaux s'ils les commettaient dans leur pays d'origine. Il s'agit de ce qu'on appelle «asymétrie définitionnelle», une pratique de plus en plus amplifiée par la mondialisation (Hall, 2013).

Dans le même ordre d'idées le fait de laisser tout le soin à chaque État de déterminer comme bon lui semble la définition et la répression des infractions relevant de la CET fait courir le risque que les États élaborent des définitions d'infractions qui varient d'un pays à l'autre, ce qui peut compliquer la tâche d'extradition (UNEP, 2013). De plus, la législation relative à la CET adoptée isolément a le potentiel d'être obscure, contradictoire et sans soutien populaire (Wright, 2011).

Pour les États comme le Canada qui fournissent des efforts notables afin de réprimer la CET, le manque de rigueur des lois fait que des peines d'emprisonnement sont très rares. Sur une période de deux ans de 2012 à 2013, seules deux personnes ont été condamnées à une peine maximale d'emprisonnement de deux mois et trois mois respectivement, ce qui est loin d'entraîner la dissuasion attendue de toute peine étant donné la nature lucrative de la CET. Par ailleurs, très peu de personnes sont condamnées pour avoir importé des substances toxiques au Canada. Selon les données apparaissant sur le site Internet du ministère de l'Environnement, sur une période de deux années, seule une entreprise a été condamnée en 2013 à une amende de 17500 \$ alors qu'on estime que le commerce illégal des déchets toxiques entre le Canada et les États-Unis génère des profits annuels de près de 2,5 millions de dollars (Elliot, 2012). L'entreprise de recyclage a plaidé coupable d'avoir importé des batteries au lithium usagées dont la quantité excédait de $68 \%$ la limite permise (Environnement Canada, 2013).

La pratique canadienne est symptomatique de ce qui se fait ailleurs dans le monde en matière de lutte contre la CET. Au sein de l'Union européenne par exemple, on rapporte un taux moins élevé de poursuites relatives à la CET par rapport aux autres segments de la criminalité organisée: entre 1992 et 2003, Fröhlich rapporte 122 cas seulement (Fröhlich, 2003). Même en ce qui concerne ces rares cas, la peine est jugée inadéquate pour dissuader les contrevenants, encore moins 
détruire les réseaux criminels organisés (Wright, 2011). En Australie par ailleurs, les crimes contre l'environnement sont sanctionnés par des amendes moins importantes puisqu'on les assimile aux dommagesintérêts de droit civil ou aux sanctions administratives (White, 2011).

La CET implique des réseaux criminels à ramifications longues qui se cachent souvent derrière des compagnies écrans dont le siège social est généralement situé dans des paradis fiscaux afin de faciliter le blanchiment des fonds illégalement obtenus (Elliot, 2012). Une telle sophistication de la criminalité est bien évidemment hors de portée de la capacité législative et policière d'un seul État et exige la combinaison des efforts de plusieurs pays, laquelle n'est malheureusement pas souvent au rendez-vous en l'absence de traités qui le prévoient expressément. L'Office des Nations Unies contre la drogue et le crime estime que les réseaux criminels opérant le trafic illégal de la faune, de la flore, des déchets électroniques et des substances appauvrissant la couche d'ozone fonctionnent à partir des différents continents en assurant la connexion entre les sources d'approvisionnement les moins chères et les points d'écoulement les plus offrants (ONUDC, 2012b). En matière de commerce illégal de l'ivoire par exemple, il est rapporté que depuis 2009, le Kenya, la Tanzanie, le Togo et la Côte d'Ivoire constituent des points à partir desquels les criminels embarquent leurs produits, alors que la Chine et la Thaillande constituent les points de destination, que différents pays servent de points de transit importants, à savoir la Malaisie, le Vietnam, Hong Kong, l'Indonésie, l'Espagne, le Sri Lanka, la Turquie et les Émirats arabes unis (TRAFFIC, 2013).

En dehors du manque de législation efficace, plusieurs pays faisant face à la CET manquent d'institutions, de technologies et de ressources tant humaines que matérielles à même de relever le défi posé par le phénomène (Wright, 2011). En effet, la lutte contre la CET est différente de la lutte contre la criminalité ordinaire compte tenu de son haut niveau d'organisation et de la puissance politique, financière et militaire de leurs auteurs et complices. Elle requiert souvent l'implication de plusieurs intervenants comme des policiers, des militaires, des agents d'enquête et de renseignement, des agents des douanes et des frontières, du corps de la justice, des scientifiques, etc., afin d'arriver à arrêter les suspects et à collecter les éléments de preuve nécessaires à obtenir la condamnation des coupables. La lutte contre la CET exige aussi du temps, des moyens financiers et de la technologie appropriée (ONUDC, 2012c). Or, un grand nombre de pays ne disposent pas nécessairement 
de toutes ces ressources indispensables pour exécuter cette tâche. Là où des institutions et du personnel qualifié existent, ils peuvent s'avérer peu équipés et peu organisés par rapport aux groupes criminels impliqués dans la CET (UICN, 2013). De plus, à l'aide de la corruption, les criminels environnementaux peuvent se chercher des complicités dans les hautes sphères politico-administratives afin d'obtenir des exemptions de contrôle à la frontière ou des documents forgés (Elliot, 2012). Dans de rares situations où les délinquants sont traduits en justice, leur puissance économique leur permet d'obtenir facilement la disparition des éléments de preuve qui auraient dû servir à leur condamnation (Elliot, 2012).

\section{Au-delà du traitement fragmentaire de la CET : imaginer une juridiction criminelle supranationale}

Devant tous les défis ci-haut mentionnés, il est donc urgent d'envisager rapidement la criminalisation des crimes environnementaux sur le plan international et la création d'une juridiction internationale pour s'attaquer à la CET. Cette proposition avait déjà été faite par le Conseil économique et social de l'ONU en 1994 (ONU, 1994). Reposant sur le bon vouloir des États qui choisissent les aspects à criminaliser, le système actuel ne parvient pas à dénoncer adéquatement la CET en tant que préoccupation internationale. Or, vu sa gravité, la CET n'a pas besoin de dépendre des législations internes pour être considérée comme grave. Dans la mesure où elle menace les fondations de la société humaine, la CET est, par sa nature même, grave et internationalement dommageable, peu importe ce que prévoient les lois des États (Tomuschat, 1996). Le système international est donc justifié à criminaliser la CET et à poursuivre les auteurs de façon autonome (ISISC, 2010; Mégret, 2011). Seule une plus grande stigmatisation provenant de l'application du droit international pénal ainsi qu'une importante dissuasion à la mesure de la gravité de la CET pourraient, sinon venir à bout du phénomène, du moins diminuer son importance et ses effets. Par ailleurs, afin d'assurer une certaine conformité avec l'actuelle pratique internationale, il serait approprié que la juridiction criminelle supranationale soit réservée à la répression de la CET commise intentionnellement ou par ignorance volontaire, et non pas celle provoquée par la simple négligence. 
Le fait que la CET n'est à ce jour régie que par le droit pénal interne, plutôt que directement par une convention internationale qui la rendrait attentatoire aux valeurs communes à l'humanité, ne permet pas de la dénoncer et d'envoyer le message que le monde n'est plus disposé à tolérer les pratiques détruisant l'environnement (Elliot, 2007). La communauté internationale doit se montrer déterminée, ferme et unie face à la CET. Il est donc nécessaire d'envoyer un signal fort que l'auteur des crimes graves contre l'environnement s'en prend aux intérêts de tous les États et que chaque État doit coopérer pour le combattre.

Étant donné que certains domaines de la CET sont liés aux activités de groupes armés ou terroristes, la communauté internationale devrait être interpellée quant à la nécessité de prendre le phénomène très au sérieux en le considérant comme une grave menace pour la paix et la sécurité internationales. Cette prise de conscience ferait peut-être germer l'idée d'amender le Statut de Rome qui a créé la Cour pénale internationale (CPI) afin d'étendre sa compétence aux crimes environnementaux. Actuellement, les crimes environnementaux que la CPI est censée réprimer se limitent à quelques atteintes à l'environnement, jugées graves, commises lors des conflits armés. En outre, pour être réprimés par la CPI, ces crimes ne doivent pas être justifiés par des nécessités militaires (Statut de Rome, art. 8-2-a-iv; 8-2-b-xiii; 8-2-exii). Autrement dit, quelle que soit leur ampleur, des crimes contre l'environnement commis pendant une guerre peuvent rester impunis s'ils sont considérés comme étant militairement justifiés. Or, les belligérants peuvent toujours réussir à justifier militairement les crimes contre l'environnement qu'ils commettent. De plus, la quasi-totalité de la CET ne se déroule pas pendant la guerre et cela n'a rien avoir avec sa gravité: des atteintes à l'environnement commises en tant de paix s'avèrent aussi nuisibles ou même plus nuisibles que celles commises pendant la guerre (Manirabona, 2011b).

Il serait donc d'un grand intérêt que la communauté internationale retourne à ses bons projets du passé qui visaient à criminaliser la CET en tant que crime international, à l'instar des crimes contre l'humanité (Drumbl, 1998). En effet, en 1976, le Projet d'articles sur la responsabilité des États proposait la création d'une infraction criminelle internationale contre l'environnement (art. 19). En 1994, le Conseil économique et social de l'ONU invitait les États à définir davantage la notion de crime international contre l'environnement (ONU, 1994). En 1996, à l'approche de l'adoption du Statut de Rome, le Projet de 
code des crimes contre la paix et la sécurité humaine (art. 29) envisageait aussi la création du crime international contre l'environnement défini comme un préjudice intentionnel et sévère à l'environnement. Mais cette proposition a été rejetée avec, entre autres, comme arguments le fait que certaines atteintes à l'environnement font déjà partie des crimes de guerre et que de toute façon, plusieurs crimes environnementaux tomberaient en deçà du niveau de gravité exigé pour que la CPI décide de poursuivre (Cassese, Gaeta \& Jones, 2002; Ezekiel, 2007). Bien sûr que si le Statut de Rome était amendé pour étendre la compétence de la CPI aux crimes environnementaux, cela exigerait de revoir la conception et l'interprétation des directives de poursuite, notamment quant à la notion de gravité. En effet, traditionnellement, la gravité s'évalue en fonction des victimes humaines et de la manière systématique ou généralisée dont les crimes ont été commis. Or, la nuisance des crimes environnementaux ne se manifeste pas nécessairement à travers les victimes humaines affectées. D'où l'intérêt de distinguer la protection des valeurs environnementales de celle des valeurs humaines à travers la reconnaissance d'un crime autonome contre l'environnement naturel, autrement appelé crime international d'écocide (Neyret, 2013), qui coexisterait avec les crimes contre l'humanité.

\section{Conclusion}

La CET est trop grave pour la laisser aux seules compétences étatiques. Il faudrait nécessairement que la communauté internationale la traite à sa juste valeur, notamment en la considérant et en la définissant comme un crime international dont la commission menace les intérêts de l'ensemble de tous les États du monde. En outre, sa nature particulièrement grave, systématique et de longue durée l'élève au rang des crimes qu'une juridiction internationale comme la Cour pénale internationale est en bonne position de réprimer. La répression de la CET par la CPI bénéficierait des avantages d'une institution fonctionnelle et qui a déjà fait preuve d'une certaine efficacité. Il est donc nécessaire que le Statut de Rome, qui a créé la CPI, soit amendé, notamment à l'article 7, et que la liste des crimes contre l'humanité inclue les crimes environnementaux.

Cependant, même s'il était effectué, l'ajout des crimes environnementaux au Statut de Rome pourrait ne pas participer au règlement de tous les problèmes relatifs à la CET dans la mesure où plusieurs contre- 
venants personnes morales ne pourraient pas être poursuivis comme le prévoit actuellement l'article 25 du Statut. Or, on sait qu'une portion importante de la CET est commise par les entreprises. Un double amendement qui toucherait les articles 7 et 25 serait donc nécessaire. Il faut toutefois souligner que le processus d'amendement du Statut de Rome pourrait s'avérer ardu compte tenu du fait que plusieurs États puissants pourraient s'y opposer comme ils l'ont déjà fait dans le passé.

D'où la proposition de créer un tribunal pénal international spécialisé en matière de CET qui appliquerait les standards propres aux questions d'environnement. Des voix se sont fait entendre récemment, entre autres pour proposer la création d'un tribunal pénal environnemental européen et d'une cour pénale internationale pour l'environnement et la santé (Charte de Bruxelles, 2014). Si la création d'un tribunal européen semble possible du fait de l'existence déjà d'un certain nombre d'instruments (Convention du Conseil de l'Europe sur la protection de l'environnement par le droit pénal du 4 novembre 1998, STE 172 et Directive européenne 2008/99/CE du 19 novembre 2008 relative à la protection de l'environnement par le droit pénal), l'établissement d'une cour pénale internationale pour l'environnement semble être une étoile inatteignable. En attendant, l'on ne peut qu'encourager les tribunaux internes à prendre le problème de la CET à bras-le-corps et d'en juger et condamner les auteurs. La nature de cette forme de criminalité requiert aussi de renforcer, d'une part, la coopération judiciaire pour tenir des procès équitables et, d'autre part, la reconnaissance mutuelle des décisions de justice pour que les jugements rendus soient effectivement exécutés. À cet égard, l'affaire Chevron, l'entreprise américaine condamnée en 2011 en Équateur à payer aux victimes de la pollution qu'elle a causée une compensation de 19 milliards de dollars est très instructive. En effet, alors que l'entreprise n'avait plus de biens saisissables en Équateur, les victimes ont cherché à faire exécuter la décision aux États-Unis où se trouve son siège social. Mais à la demande de Chevron, un tribunal new-yorkais a, en mars 2014, annulé la décision rendue en Équateur au motif que le processus à la base du jugement avait été caractérisé par la corruption.

Finalement, la prévention par des moyens non judiciaires devrait aussi être renforcée notamment à travers la sensibilisation et l'éducation des populations impliquées dans le phénomène afin de réduire l'offre et la demande des produits issus de cette criminalité. 


\section{Références}

Agence France-Presse (AFP). (2014). Afrique du Sud: plus de mille rbinocéros tués par des braconniers en 2013. Repéré à http://www.jeuneafrique.com/actu/ 20140117T153050Z20140117T153025Z/afrique-du-sud-plus-de-millerhinoceros-tues-par-des-braconniers-en-2013.html.

Agnew, R. (2012). Dire forecast: A theoretical model of the impact of climate change on crime. Theoretical Criminology 16(1), 21-42.

Amnesty International. (2004). Clouds of Injustice: Bhopal Disaster 20 Years On. London: Amnesty International.

Amnesty International. (2009). Pétrole, pollution et pauvreté dans le delta du Niger. Londres: Amnesty International. Repéré à: https://www.amnesty.org/en/ library/asset/AFR44/018/2009/fr/7c519761-8e16-4c60-b5967fc763ae7371/afr440182009fra.pdf.

Bossy D. (2012, 22 octobre). Naufrage du Prestige: 10 ans plus tard, où est passé le fioul?. Futura-Sciences.

Carnier, J. (2014, 31 janvier). Charte de Bruxelles pour une justice internationale de l'environnement. RSE magazine. Repéré à http://www.rse-magazine. com/Charte-de-Bruxelles-pour-une-justice-internationale-de-1environnement_a480.html.

Casselman, A. (2011, 20 avril). A Year After the Spill, "Unusual” Rise in Health Problems More cases of nosebleeds, coughs could be due to oil exposure. National Geographic News.

Cassese, A., Gaeta, P., \& Jones, J. R. W. D. (2002). The Rome Statute of the International Criminal Court: A Commentary, 522-23.

Clapp, J. (2005). Global Environmental Governance for Corporate Responsibility and Accountability. Global Environmental Politics, 5(3), 23-34.

Drumbl, M. A. (1998). Waging War against the World: The Need to Move from War Crimes to Environmental Crimes. Fordham International Law Journal, 22(1), 122-153.

Elliot, L. (2007). Transnational environmental crime in the Asia Pacific: an "un(der)securitized" security problem? The Pacific Review, 20(4), 499-522.

Elliot, L. (2012). Fighting Transnational Environmental Crime. Journal of International Affairs, 66(1), 87-104.

Environnement Canada. (2013). L'entreprise Toxco Waste Management Ltd. écope d'une amende de 17500 dollars pour avoir importé des matières recyclables dangereuses (Communiqué de presse, 18 avril). Ottawa.

Ezekiel, A. (2007). The Application of International Criminal Law to Resource Exploitation: Ituri, Democratic Republic of the Congo. Natural Resources Journal, 47(1), 225.

Fröhlich, T. (2003). Organised environmental crime in the EU Member States. Kassel: BfU.

Gilbert, M. J., \& Russell, S. (2002). Globalization of criminal justice in the corporate context Crime, law and social change, 38, 211-238. 
Gosling, J. (2013). The Global Response to Transnational Organized Environmental Crime, Global Initiative against Transnational Organized Crime. Repéré à http:// www.globalinitiative.net/download/global-initiative/Gosling\%20-\%20 Environmental\%20Crime.pdf

Hall, M. (2013). Environmental harm and environmental victims : scoping out a 'green victimology. International Review of Victimology, 20(1), 129-143.

INTERPOL. (2012). Environmental Crime. International Criminal Police Organization. Repéré à http://www.interpol.int/Crime-areas/Environmentalcrime/Environmental-crime.

INTERPOL. (2013). Guide to Carbon Trading Crime. Lyon: Interpol.

INTERPOL. (2014). Le Comité sur la criminalité de l'environnement, Groupe de travail sur la criminalité liée à la pollution. Repéré à http://www.interpol.int/ fr/Crime-areas/Environmental-crime/Environmental-Compliance-andEnforcement-Committee/Pollution-Crime-Working-Group.

IRIN. (2013). Environmental Crimes Increasingly Linked to Violence, Insecurity. Irinnews. Repéré à http://www.irinnews.org/printreport.aspx? reportid $=98872$.

ISISC. (2010, 12-19 avril). The Protection of the Environment through Criminal Law (A/CONF.213/NGO/10). Statement submitted at the 12th United Nations Congress on Crime Prevention and Criminal Justice, Salvador, Brazil. Repéré à https://cms.unov.org/llsulinkbase/contenttree.aspx?nodeID $=3406$.

Jasparro, C., \& Taylor, J. (2008). Climate change and regional vulnerability to transnational security threats in Southeast Asia. Geopolitics, 13(2), 232-256.

Lynch, M., Burns, R., \& Stretesky, P. (2010). Global warming and statecorporate crime: The politicization of global warming under the Bush administration. Crime, Law and Social Change, 54, 213-239.

Manirabona, A. (2011a). Entreprises multinationales et criminalité environnementale transnationale: Applicabilité du droit pénal canadien. Cowansville: Éditions Yvon Blais.

Manirabona, A. (2011b). L'affaire Trafigura: Vers la répression de graves atteintes environnementales en tant que crimes contre l'humanité? Revue de droit international et de droit comparé, 4, 535-576.

Mares, D. (2010). Criminalizing Ecological Harm: Crimes Against Carrying Capacity and the Criminalization of Eco-Sinners. Critical Criminology, 18, 279-293.

Mason, M. (2008). The governance of transnational environmental harm: addressing new modes of accountability/responsibility. Global Environmental Politics, 8(3), 8-240.

Mégret, F. (2011). The Problem of an International Criminal Law of the Environment. Columbia Journal of Environmental law, 36(2), 195-257.

Neyret, L. (2013). La transformation du crime contre 1'humanité. Dans M. Delmas-Marty, I. Fouchard, E. Fronza \& L. Neyret (Éds), Le crime contre l'bumanité (2 edition) (pp.80-116). Paris: Presses Universitaires de France. 
O'Hear, M. M. (2004). Sentencing the Green-Collar Offender: Punishment, Culpability, and Environmental Crime. Journal of Criminal Law er Crimino$\log y$, 95(1), 133.

Organisation for Economic Cooperation and Development (OCDE). (2012). Illegal Trade in Environmentally Sensitive Goods. OECD Trade Policy Studies. Repéré à http://dx.doi.org/10.1787/9789264174238-en.

Organisation des Nations Unies (ONU), Conseil Économique et Social. (1994). The role of criminal law in the protection of the environment (Résolution 1994/15). New York: ONU. Repéré à http://www.un.org/documents/ecosoc/res/ 1994/eres1994-15.htm.

Organisation des Nations Unies (ONU), Secrétariat général. (1995). Results of the supplement to the Fourth United Nations Survey on Crime Trends and Operations of Criminal Justice Systems, on transnational crime: interim report, (A/CONF.169/ 15/Add.1 (1995)). New York: ONU.

Organisation des Nations Unies (ONU), Conseil de sécurité. (2013). Report of the Secretary-General on the activities of the United Nations Regional Office for Central Africa and on the Lord's Resistance Army-affected areas. New York: ONU.

Office des Nations Unies contre la Drogue et le Crime (ONUDC). (2012a). Digest of organized crime cases. A compilation of cases with commentaries and lessons learned. Repéré à https://www.unodc.org/documents/organized-crime/ EnglishDigest_Final301012_30102012.pdf.

Office des Nations Unies contre la Drogue et le Crime (ONUDC). (2012b, 15-19 octobre). Report of the Secretariat on technical assistance provided to States in the application of the United Nations Convention against Transnational Organized Crime to new forms and dimensions of transnational organized crime (CTOC/ COP/2012/7). Report submitted at the $6^{\text {th }}$ session of Conference of the Parties to the United Nations Convention against Transnational Organized Crime, Vienne, Autriche. Repéré à http://www.unodc.org/unodc/treaties/ CTOC/CTOC-COP-session6.html.

Office des Nations Unies contre la Drogue et le Crime (ONUDC). (2012c). Digest of organised crime cases: A compilation of cases with commentaries and lessons learned. Vienne: United Nations Office on Drugs and Crime. Repéré à http://www.unodc.org/documents/organized-crime/EnglishDigest_Final 301012_30102012.pdf.

Oxford Economics. (2010). Potential Impact of the Gulf oil spill on tourism. Report Prepared for the U.S. Travel Association. Oxford: Oxford Economics.

Salo, R. S. (2003). When the Logs Roll Over: The Need for an InternationalConvention CriminalizingInvolvement in the Global Illegal Timber Trade. Georgetown International Environmental Law Review, 16, 137-39.

Scanlon, J. (2012). Ivory and Insecurity: The Global Implications of Poaching in Africa. CITES. Repéré à http://www.cites.org/eng/news/SG/2012/ 20120525_SG_US-Senate_testimony.php

Skinnider, E. (2011). Victims of Environmental Crime-Mapping the Issues. Vancouver: The International Centre for Criminal Law Reform and Criminal Justice Policy. 
Tomuschat, C. (1996). Document on Crimes Agdinst the Environment (vol. II). Yearbook of the International Law Commission 15. New York: ONU.

TRAFFIC. (2013). New figures reveal poaching for the illegal ivory trade could wipe out a fifth of Africa's Elephants over next decade. Repéré à http://www.traffic. org/home/2013/12/2/new-figures-reveal-poaching-for-the-illegal-ivorytrade-coul.html.

Union Internationale pour la Conservation de la Nature. (2013, 2-4 décembre). Mesures d'urgence. Présentées lors du Sommet de l'Éléphant d'Afrique, Gaborone, Botswana. Repéré à https://cmsdata.iucn.org/downloads/sommet_ de_1_elephant_d_afrique_les_mesures_d_urgence_finale_3_dec_2013. pdf.

United Nations Environment Program (UNEP). (2013). Transnational Environmental Crime - A Common Crime in Need of Better Enforcement. Nairobi: UNEP. Repéré à http://na.unep.net/geas/getUNEPPageWithArticleIDScript.php? article_id $=95$.

United Nations Environment Programme (UNEP) \& Interpol. (2012). Green Carbon, Black Trade: Illegal Logging, Tax Fraud and Laudering in the World's Tropical Forests. Nairobi: UNEP.

White, R. (2010). Prosecution and Sentencing in Relation to Environmental Crime: Recent Socio-Legal Developments. Crime, Law and Social Change, 53(4), 365-381.

White, R. (2011). Transnational Environmental Crime: Toward an Eco-global Criminology. New York: Routledge

Williams, C. (1996). An environmental victimology. Social Science, 23 (1), $16-40$.

Wright, G. (2011). Conceptualising and combating transnational environmental crime. Trends in Organized Crime, 14, 332-346.

\section{Lois internationales}

Convention des Nations Unies contre la criminalité transnationale organisée. Adoptée par la résolution 55/25 de l'Assemblée générale. [15 novembre 2000].

Convention sur la Conservation des Espèces Migratrices appartenant à la faune sauvage (CMS). [23 Juin1979]. 1651 UNTS 333.

Convention sur le Commerce International des Espèces de Faune et de Flore Sauvages Menacées d'Extinction (CITES). [3 Mars 1973]. 993 U.N.T.S. 243.

Statut de Rome de la Cour pénale international. [17 juillet 1998].

\section{Jurisprudence}

Choc c. Hudbay Minerals Inc., 2013 ONSC 1414 
ABSTRACT - This paper proposes the way in which the international community should address transnational environmental crime. To attain this goal, the author first defines the phenomenon and discusses its scope. The approach consists of understanding this form of crime so that interested parties can envisage appropriate means to combat it. The article highlights the current absence of adequate means to combat this grave phenomenon and claims that the magnitude and characteristics of transitional environmental crime merit the mobilization of the international community as a whole. Specifically, the author argues that, apart from strengthening control mechanisms at the national level and improving judicial cooperation and mutual recognition of judicial decisions, creating an international criminal tribunal for the environment would be an ideal option.

KEYWORDS • Environment, crimes, transnational, harm, gravity.

RESUMEN - El presente estudio propone la manera en la que la comunidad internacional debería abordar la criminalidad medioambiental transnacional. Para ello, el autor, primeramente, define este fenómeno y discute sobre su magnitud. El enfoque consiste en hacer entender esta forma de criminalidad, con el fin de inducir a las partes interesadas a considerar los medios apropiados para combatirla. Luego, el artículo recuerda la inexistencia de medios de lucha que estén a la altura del grado de gravedad de esta plaga y sostiene que, la importancia y las características de ésta, merecen la movilización de toda la comunidad internacional. Específicamente, el autor sostiene que, más allá del refuerzo de los mecanismos de lucha en el plano nacional, de la mejora de la cooperación judicial, y del reconocimiento mutuo de las decisiones de la justicia, la creación de un tribunal penal internacional del medioambiente sería una opción ideal.

PALABRAS-CLAVE • Medioambiente, crímenes, transnacional, daño, gravedad. 